\title{
Hypomaturation Amelogenesis Imperfecta due to WDR72 Mutations: A Novel Mutation and Ultrastructural Analyses of Deciduous Teeth
}

\author{
W. El-Sayed ${ }^{a, b} \quad$ R.C. Shore ${ }^{a} \quad$ D.A. Parry ${ }^{b} \quad$ C.F. Inglehearn ${ }^{b} \quad$ A.J. Mighell ${ }^{a} b$ \\ ${ }^{a}$ Leeds Dental Institute and ${ }^{\mathrm{b}}$ Leeds Institute of Molecular Medicine, University of Leeds, Leeds, UK
}

\section{Key Words}

Amelogenesis imperfecta - WDR72 - Enamel • Teeth •

Scanning electron microscopy

\begin{abstract}
Background: Mutations in WDR72 have been identified in autosomal recessive hypomaturation amelogenesis imperfecta (Al). Objective: to describe a novel WDR72 mutation and report the ultrastructural enamel phenotype associated with a different WDR72 mutation. Methods: A family segregating autosomal recessive hypomaturation Al was recruited, genomic DNA obtained and WDR72 sequenced. Four deciduous teeth from one individual with a previously published WDR72 mutation, extracted as part of clinical care, were subjected to scanning electron microscopy, energydispersive X-ray analysis and transverse microradiography. Results: A novel homozygous nonsense mutation, R897X, was identified in WDR72 in a family originating from Pakistan. Ultrastructural analysis of enamel from the deciduous teeth of an Al patient with the WDR72 mutation S783X revealed energy-dispersive $\mathrm{X}$-ray analysis spectra with normal carbon and nitrogen peaks, excluding retention of enamel matrix protein. However, transverse microradiography values were significantly lower for affected teeth when compared to normal teeth, consistent with reduced mineralisation. On scanning electron microscopy the enamel rod form observed was normal, yet with inter-rod enamel more prominent than in controls. This appearance was unaltered fol-
\end{abstract}

lowing incubation with either $\alpha$-chymotrypsin or lipase. Conclusions: The novel WDR72 mutation described brings the total reported WDR72 mutations to four. Analyses of deciduous tooth enamel in an individual with a homozygous WDR72 mutation identified changes consistent with a late failure of enamel maturation without retention of matrix proteins. The mechanisms by which intracellular WDR72 influences enamel maturation remain unknown.

Copyright $\odot 2010$ S. Karger AG, Basel

\section{Introduction}

Enamel is unique among human biomineralised tissues and when formed normally can last a lifetime without any significant capacity for repair. It is approximately $95 \%$ mineral content by weight [Smith, 1998] and primarily consists of large hydroxyapatite $\left(\mathrm{Ca}_{10}\left[\mathrm{PO}_{4}\right]_{6}[\mathrm{OH}]_{2}\right)$

\section{Abbreviations used in this paper}

AI amelogenesis imperfecta

EDX energy dispersive X-ray analysis

KLK4 kallikrein 4

MMP20 matrix metalloproteinase 20

SEM scanning electron microscopy

TMR transverse microradiography

\section{KARGER}

(๑) 2010 S. Karger AG, Basel

Fax +41 613061234

E-Mail karger@karger.ch

www.karger.com
Accessible online at: www.karger.com/cto
Dr. Alan J. Mighell

Department of Oral Medicine, Leeds Dental Institute

Clarendon Way

Leeds LS2 9LU (UK)

Tel. +44 113343 6121, Fax +44113343 6165, E-Mail a.j.mighell@leeds.ac.uk 
crystals [Nylen et al., 1963; Daculsi and Kerebel, 1978]. A key to enamel strength is the highly ordered orientation of hydroxyapatite crystals within enamel rods. Each enamel rod reflects the pathway taken by an ameloblast [Skobe, 2006]. These epithelial-derived cells are responsible for secretion of the enamel protein matrix necessary for mineralisation and then its controlled removal as the enamel matures to its final form [Porto et al., 2009].

Amelogenesis, the process of enamel formation, can be considered to occur in three stages: secretory, transition and maturation [Warshawsky and Smith, 1974; Smith and Nanci, 1995]. As secretory stage ameloblasts retreat towards the eventual tooth surface they produce enamel matrix that includes amelogenin [Yang et al., 2010], enamelin [Al-Hashimi et al., 2009] and ameloblastin [Chun et al., 2010]. The matrix immediately begins to mineralise [Kirkham et al., 1988; Cuisinier et al., 1992; Smith, 1998] and this process is promoted further via ameloblast-mediated enamel matrix processing, in which matrix metalloproteinase 20 (MMP20) plays a critical role [Iwata et al., 2007]. Kallikrein 4 (KLK4), a serine protease enzyme, is functional later during the maturation stage as hydroxyapatite crystal growth is completed and virtually all organic matrix is removed [Simmer and $\mathrm{Hu}$, 2002; Simmer et al., 2009]. Ameloblasts then undergo apoptosis at the external enamel surface prior to tooth eruption [Tsuchiya et al., 2009].

Amelogenesis imperfecta (AI) is a genetically and clinically heterogeneous group of inherited conditions typically characterised by generalised enamel defects of both primary and permanent dentitions [Witkop, 1988; Aldred et al., 2003]. The clinical phenotype is influenced by the stage of amelogenesis predominantly affected, although clinical classification can be difficult. Hypoplastic forms of AI are due to defects in the secretory stage leading to diminished volumes of enamel matrix protein, which in turn result in very thin enamel. By contrast, hypocalcified and hypomaturation forms of $\mathrm{AI}$ are characterised by near-normal volumes of enamel matrix that typically is not processed and removed appropriately, leading to failure of normal biomineralisation [Witkop, 1988; Ng and Messer, 2009]. In hypomaturation AI the opaque, discoloured enamel typically chips away from the supporting dentine, whereas in hypocalcified AI the enamel is soft enough to scrape away with a hand instrument [Witkop, 1988]. These two forms of AI are sometimes collectively referred to as hypomineralised AI, reflecting the difficulties in distinguishing between ill-defined phenotypes, especially where teeth have been subjected to post-eruption changes.
Mutations in 6 genes have been shown to cause AI to date. These include the enamel matrix protein encoding genes AMELX (MIM 301200) [Lagerström et al., 1991; Barron et al., 2010] and ENAM (MIM 608563) [Rajpar et al., 2001; Hart et al., 2003] as well as the enamel matrixmodifying protease genes MMP20 (MIM 612529) [Kim et al., 2005; Lee et al., 2010] and KLK4 (MIM 603767) [Hart et al., 2004]. In addition, mutations in FAM83H (MIM 130900), a gene of unknown function that encodes an intracellular protein, are a frequent cause of autosomal dominant hypocalcified AI [Kim et al., 2008; Lee et al., 2008; El-Sayed et al., 2010]. Finally, we reported three different WDR72 mutations as a novel cause of autosomal recessive hypomaturation $\mathrm{AI}$ in families originating from either Pakistan or Oman [El-Sayed et al., 2009]. WDR72 is an intracellular protein with a predicted $\beta$-propeller structure expected to mediate reversible protein-protein interactions. Its functions remain unknown and its discovery has opened a new area of research in enamel biomineralisation.

In this study we report a novel homozygous mutation in exon 15 of WDR72, causing hypomaturation $\mathrm{AI}$ in a further consanguineous Pakistani family, underlining the importance of WDR72 mutations as a cause of autosomal recessive hypomaturation AI. In addition we report the first ultrastructural analyses of deciduous teeth from a patient in a previously reported AI family with a homozygous mutation in WDR72.

\section{Materials and Methods}

Subjects

A consanguineous family (P6) of Pakistani origin was ascertained, in which 2 sisters had hypomaturation AI. Peripheral blood samples were obtained from 1 affected individual and genomic DNA was prepared by a conventional salting method. Four deciduous molar teeth affected by AI that had been extracted for clinical reasons were obtained from an individual in a previously reported family (P2) with homozygous c.2348C $>$ G; p.S783X mutation [El-Sayed et al., 2010]. Samples were obtained with informed consent in a process approved by the Leeds (West) NHS Trust Ethics committee.

WDR72 Mutation Analysis

All 19 coding exons and exon/intron boundaries of the WDR72 gene were PCR amplified using oligonucleotide primers described by El-Sayed et al. [2009]. PCR products were purified using EXOSAP enzyme. Purified products were sequenced using the Big Dye terminator Kit v.3.1 (Applied Biosystems, Forster City, Calif., USA) and size fractionated on an ABI 3130 XL DNA analyser. The sequence produced was analysed using the ABI Prism sequence Analyser and SeqScape software packages (Applied Biosystems).

Cells Tissues Organs 2011;194:60-66 
Scanning Electron Microscopy, Energy-Dispersive X-Ray

Spectroscopy and Transverse Microradiography

Standard methods were used for preparation of tooth sections $(100 \mu \mathrm{m})$ from deciduous teeth. These were then investigated by scanning electron microscopy (SEM), energy-dispersive X-ray spectroscopy (EDX) and transverse microradiography (TMR) [Shore et al., 2002; Barron et al., 2008; El-Sayed et al., 2010]. Microstructural analysis was undertaken using a Jeol 35 SEM fitted with the Deben Genie upgrade (Deben Engineering, Debenham, UK). EDX elemental analysis was performed using a detector fitted with an ultrathin window and driven by WinEDX 3 software (Thomson Scientific, Carlton, Vic., Australia). TMR involved sampling across sections from 4 affected and 2 control teeth at a minimum of 10 different points each within the enamel. Analysis was done using Inspektor TMR software version 20.0.27.16, 2000 (Inspektor Research Systems, Amsterdam, The Netherlands). For enzyme digestion, scanned sections were re-polished and re-etched with $35 \%$ phosphoric acid for $15 \mathrm{~s}$. Sections were incubated in either $\alpha$-chymotrypsin (C5088-2MG; Sigma, Dorset, UK) or lipase (Sigma) as described previously [Shore et al., 2002].

\section{Results}

\section{WDR72 Mutation and Clinical Phenotype}

The clinical phenotype observed in the proband and her sister from the new AI family (P6) was consistent with AI with extensive post-eruptive enamel loss. No other health problems segregated with AI. The dentitions in both sisters were heavily restored with the remaining enamel pigmented and predominantly yellow/brown in colour (fig. 1a). Serial dental radiographs taken over many years were reviewed for both sisters and were characterised by features consistent with hypomaturation AI (fig. 1b-d). The pattern of inheritance was consistent with autosomal recessive inheritance (fig. le).

Sequencing all the exons and intron-exon boundaries of the WDR72 gene identified a nonsense mutation (c.2728C>T; p.R897X) in exon 15 of WDR72 (forward and reverse strands) in the affected proband (fig. 1e).

\section{Ultrastructural Analyses of Deciduous Teeth}

TMR sampling of teeth from the Pakistani family (P2) with a previously identified mutation in WDR72 [E]Sayed et al., 2010] revealed a reduction in the mean mineral density percentage in affected enamel $(37.9 \% \pm$ $2.83 \%)$ compared to control enamel $(76.9 \% \pm 3.81 \%)$. This reduction is statistically significant $(\mathrm{p}<0.001)$. Elemental analysis by EDX demonstrated that the ratio of carbon:oxygen and calcium:phosphorous were indistinguishable from control enamel with no nitrogen present (fig. 2a, b). This excludes the possibility that there is re- tained protein in WDR72 mutant teeth as has been shown in other forms of AI.

SEM examination of enamel from affected teeth identified obvious enamel rods across the enamel width available for examination (fig. 2c-e). However, in affected enamel the boundaries (inter-rod enamel) between individual enamel rods were more obvious and of a different electron density to those observed in normal enamel. There also appeared to be reduced decussation of the layers of enamel rods that give rise to the Hunter-Schreger bands observed in normal enamel. The SEM appearances of affected and control enamel were unaltered after incubation of the sections with the $\alpha$-chymotrypsin or lipase (data not presented).

\section{Discussion}

The p.R897X mutation is the fourth WDR72 mutation described in AI patients. Like the three previously observed mutations (p.S783X, p.W978X and p.S953VfsX20), it lies within exons $14-16$ of a 19-exon gene, which may suggest a degree of clustering towards the carboxy-terminus, downstream of the WD40 domains. The mechanism by which WDR72 nonsense mutations result in hypomaturation $\mathrm{AI}$ is unknown, but the lack of missense mutations suggests a null phenotype due to mRNA nonsensemediated decay rather than production of stable truncated proteins.

EDX is a sensitive technique for identification of retained organic material in enamel [Shore et al., 2002; Barron et al., 2008; El-Sayed et al., 2010]. When organic material is retained, incubation with either $\alpha$-chymotrypsin or lipase can be informative and reveal the enamel ultrastructure in greater detail [Shore et al., 2002]. In this study the absence of retained enamel matrix protein in affected enamel indicates that ameloblasts are able to direct degradation and removal of enamel matrix in a manner similar to that in normal teeth. MMP20 and KLK4, which are expressed predominantly by secretory and maturation-stage ameloblasts, respectively, are the two proteases critical to this process of protein degradation and removal. Mutations in their respective genes are recognised to result in an autosomal recessive hypomaturation AI clinical phenotype with reduced enamel mineralisation evident on dental radiography [Hart et al., 2004; Kim et al., 2005; Ozdemir et al., 2005; Papagerakis et al., 2008; Simmer et al., 2009]. Characterisation of mouse models null for either Mmp20 or Klk4 confirmed some inappropriate retention of enamel matrix proteins 

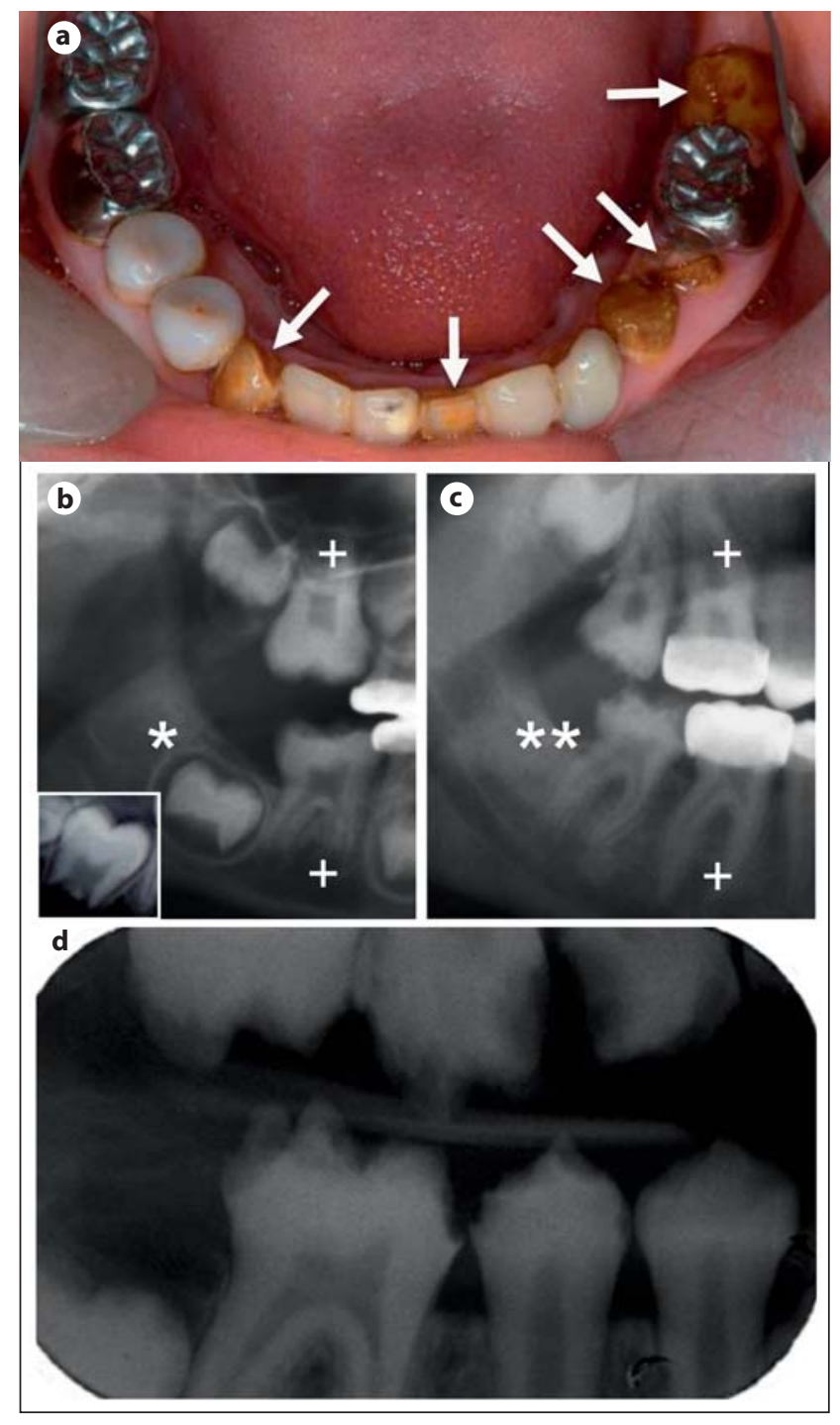

Fig. 1. Clinical and radiographic phenotype, pedigree and novel WDR72 mutation. a Heavily restored lower dentition of the proband's sister aged 18 years. The visible enamel of teeth, including where restorations have failed, has a yellow/brown discolouration (arrows). b-d Radiographs of two affected individuals. Details from panoramic radiographs taken of the proband's sister when aged 8 years (b) and 20 years (c). The developing lower right second molar tooth $\left(^{*}\right)$ has a normal crown morphology in $\mathbf{b}$, but without the expected contrast in radiodensity observed in someone without AI (inset). The same tooth $\left(^{* *}\right)$ approximately 8 years

[Simmer et al., 2009; Wright et al., 2009]. Absence of residual proteins in enamel in this study rules out WDR72 as a critical upstream intracellular regulator of MMP20 or KLK4 expression or secretion from ameloblasts.

In health enamel rods abut each other with a thin layer of inter-rod enamel that is characterised by different
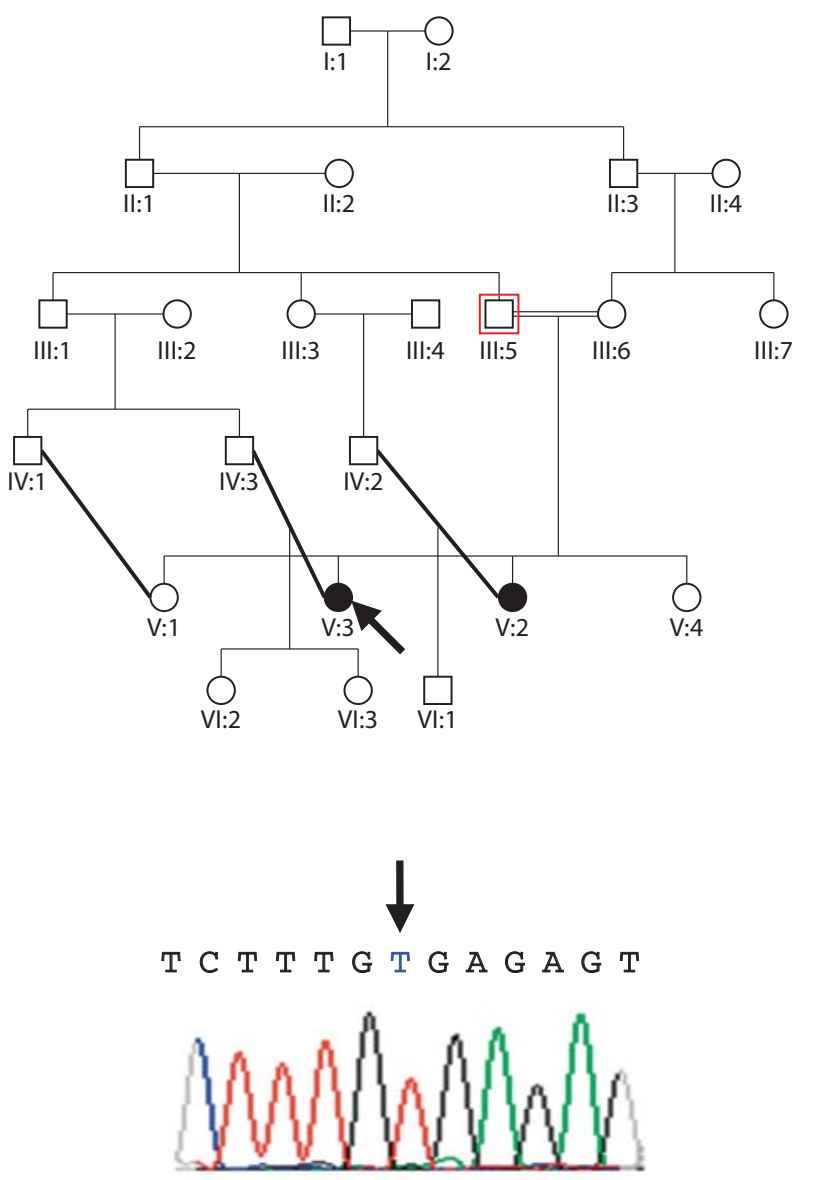

after eruption and restoration failure is characterised by considerable enamel loss consistent with early functional failure. Both the upper and lower first permanent molar teeth $(+)$ have required restoration with metal crowns due to functional failure. $\mathbf{d}$ A dental bitewing radiograph of the proband taken aged 12 years illustrating the typical post-eruptive pattern of enamel loss that starts soon after tooth eruption into the mouth. e The pedigree confirmed consanguinity (proband marked with arrow) with confirmation of the c.2728C $>$ T mutation.

orientation of hydroxyapatite crystals rather than a different composition [Boyde, 1989]. The abnormal appearance of the inter-rod enamel observed in this study may represent disruption to this late stage of enamel maturation. Little is known about the control of inter-rod enamel formation. Variations in inter-rod enamel are recog- 

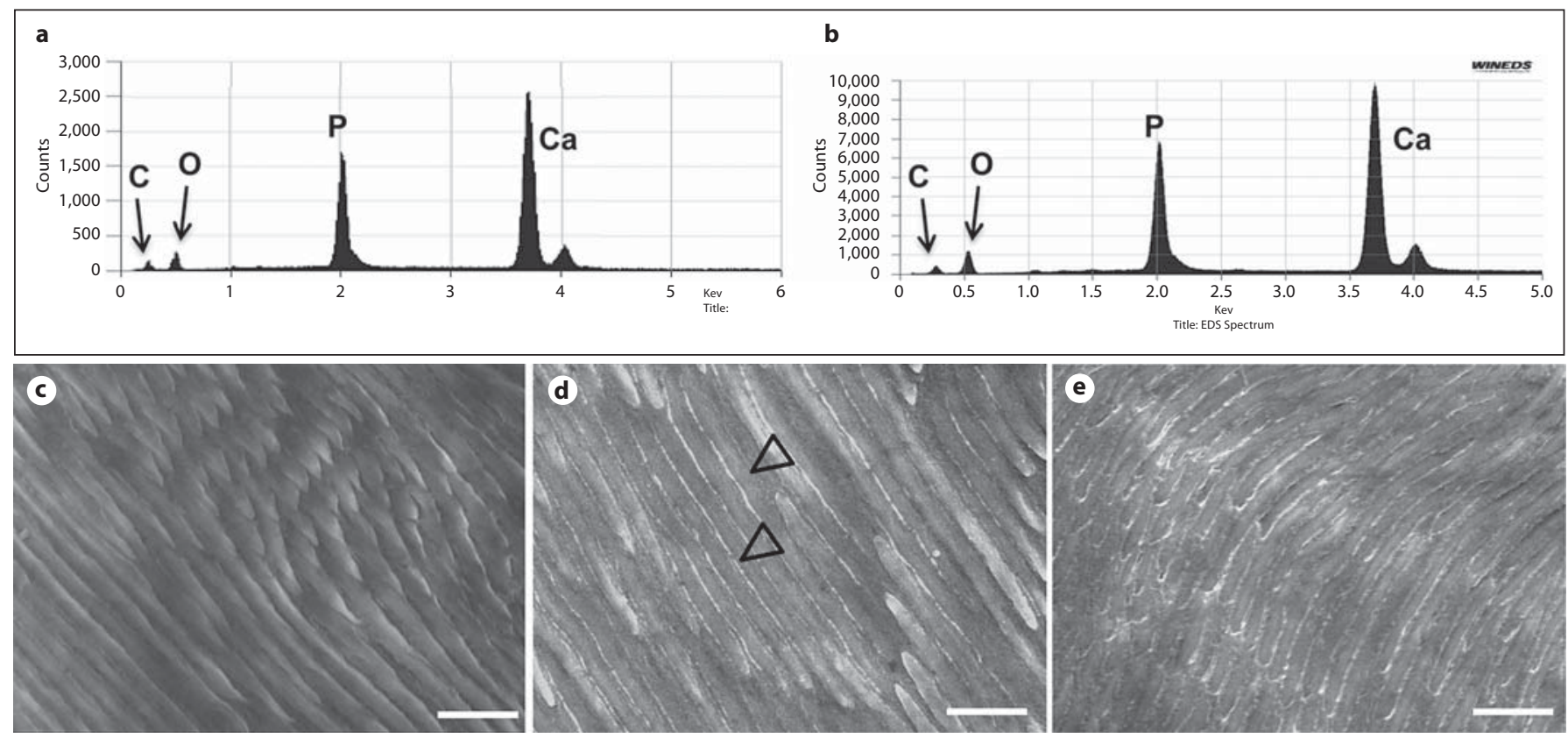

Fig. 2. Ultrastructural phenotyping of deciduous teeth. a, b EDX spectra: the C:O and Ca:P ratios were indistinguishable between control (a) and autosomal recessive hypomaturation AI (b) enamel with no $\mathrm{N}$ peak (between the $\mathrm{C}$ and $\mathrm{O}$ peaks) observed in either sample. c-e SEM of control (c) and AI-affected (d, e) enamel. The AI enamel rods are grossly normal but the inter-rod enamel is more obvious (pale lines marked with arrow heads) in the affected enamel compared to the controls. There is an apparent lack of enamel rod decussation in the affected enamel whereas in the control enamel there is different rod long axis orientation in the upper compared to the lower part of the image. Scale bars $=20 \mu \mathrm{m}$.

Mineralisation requires the delivery of relevant ions to the maturing crystals. In part this is mediated via active $\mathrm{Ca}^{2+}$ efflux/extrusion from ameloblast sodium-calcium exchangers [Okumura et al., 2010]. Maturation stage ameloblasts secrete proteins such as amelotin and odontogenic ameloblast-associated protein at the interface between ameloblasts and maturing enamel, and these are believed to play important roles in enamel maturation [Moffatt et al., 2008; Gao et al., 2010]. Control of the secretory functions of maturation stage ameloblasts remains unknown. WDR7, the closest homologue of WDR72, is involved in $\mathrm{Ca}^{2+}$-dependent vesicle exocytosis, raising the possibility that WDR72 may have a similar role in amelogenesis [Nagano et al., 2002; Coleman and Bykhovskaia, 2009].

The data presented are primarily consistent with disruption to late amelogenesis. However, the apparent loss of enamel rod decussation raises the possibility of a fundamental alteration to the way that adjacent ameloblasts interact with each other during secretion. Any lack of prism decussation would be likely to have a detrimental effect on the physical properties of the final enamel, but this observation requires confirmation in further samples. 
In conclusion, WDR72 mutations are a significant cause of autosomal recessive hypomaturation AI with a detrimental impact on the late stages of enamel maturation in amelogenesis. Elucidating the functions of WDR72 can be expected to provide novel and important insight into biomineralisation.

\section{Acknowledgements}

The authors thank the families for their participation. This work was funded by the Egyptian Government (W.E.-S.) and the Wellcome Trust (grant No. 082448 and 075945).

\section{References}

1 Al-Hashimi, N., J.Y. Sire, S. Delgado (2009) Evolutionary analysis of mammalian enamelin, the largest enamel protein, supports a crucial role for the $32-\mathrm{kDa}$ peptide and reveals selective adaptation in rodents and primates. J Mol Evol 69: 635-656.

2 Aldred, M.J., R. Savarirayan, P.J. Crawford (2003) Amelogenesis imperfecta: a classification and catalogue for the 21st century. Oral Dis 9: 19-23.

3 Barron, M.J., S.J. Brookes, C.E. Draper, D. Garrod, J. Kirkham, R.C. Shore, M.J. Dixon (2008) The cell adhesion molecule nectin-1 is critical for normal enamel formation in mice. Hum Mol Genet 17: 3509-3520.

4 Barron, M.J., S.J. Brookes, J. Kirkham, R.C. Shore, C. Hunt, A. Mironov, N.J. Kingswell, J. Maycock, C.A. Shuttleworth, M.J. Dixon (2010) A mutation in the mouse Amelx trityrosyl domain results in impaired secretion of amelogenin and phenocopies human Xlinked amelogenesis imperfecta. Hum Mol Genet 19: 1230-1247.

5 Boyde, A. (1989) Enamel; in Oksche A, Vollrath L (eds): Handbook of Microscopic Anatomy. Berlin, Springer-Verlag, pp 309-473.

-6 Chun, Y.P., Y. Yamakoshi, F. Yamakoshi, M. Fukae, J.C. Hu, J.D. Bartlett, J.P. Simmer (2010) Cleavage site specificity of MMP-20 for secretory-stage ameloblastin. J Dent Res 89: 785-790.

7 Coleman, W.L., M. Bykhovskaia (2009) Rab3a-mediated vesicle recruitment regulates short-term plasticity at the mouse diaphragm synapse. Mol Cell Neurosci 41: 286-296.

8 Cuisinier, F.J., P. Steuer, B. Senger, J.C. Voegel, R.M. Frank (1992) Human amelogenesis. I: high resolution electron microscopy study of ribbon-like crystals. Calcif Tissue Int 51: 259-268.

9 Daculsi, G., B. Kerebel (1978) High-resolution electron microscope study of human enamel crystallites: size, shape, and growth J Ultrastruct Res 65: 163-172.

10 El-Sayed, W., D.A. Parry, R.C. Shore, M. Ahmed, H. Jafri, Y. Rashid, S. Al-Bahlani, S. Al Harasi, J. Kirkham, C.F. Inglehearn, A.J. Mighell (2009) Mutations in the $\beta$ propeller WDR72 cause autosomal-recessive hypomaturation amelogenesis imperfecta. Am J Hum Genet 85: 699-705.
11 El-Sayed, W., R.C. Shore, D.A. Parry, C.F Inglehearn, A.J. Mighell (2010) Ultrastructural analyses of deciduous teeth affected by hypocalcified amelogenesis imperfecta from a family with a novel Y458X FAM83H nonsense mutation. Cells Tissues Organs 191: 235-239.

12 Gao, Y., W. Wang, Y. Sun, J. Zhang, D. Li, Y. Wei, T. Han (2010) Distribution of amelotin in mouse tooth development. Anat Rec 293: 135-140.

13 Hart, P.S., T.C. Hart, M.D. Michalec, O.H. Ryu, D. Simmons, S. Hong, J.T. Wright (2004) Mutation in kallikrein 4 causes autosomal recessive hypomaturation amelogenesis imperfecta. J Med Genet 41: 545-549.

14 Iwata, T., Y. Yamakoshi, J.C. Hu, I. Ishikawa, J.D. Bartlett, P.H. Krebsbach, J.P. Simmer (2007) Processing of ameloblastin by MMP 20. J Dent Res 86: 153-157.

15 Kim, J.W., S.K. Lee, Z.H. Lee, J.C. Park, K.E. Lee, M.H. Lee, J.T. Park, B.M. Seo, J.C. Hu, J.P. Simmer (2008) FAM83H mutations in families with autosomal-dominant hypocalcified amelogenesis imperfecta. Am J Hum Genet 82: 489-494.

16 Kim, J.W., J.P. Simmer, T.C. Hart, P.S. Hart, M.D. Ramaswami, J.D. Bartlett, J.C. Hu (2005) MMP-20 mutation in autosomal recessive pigmented hypomaturation amelogenesis imperfecta. J Med Genet 42: 271-275.

17 Kirkham, J., C. Robinson, J.A. Weatherell, A. Richards, O. Fejerskov, K. Josephsen (1988) Maturation in developing permanent porcine enamel. J Dent Res 67: 1156-1160.

18 Lacruz, R.S., A. Nanci, I. Kurtz, J.T. Wright, M.L. Paine (2010) Regulation of $\mathrm{pH}$ During Amelogenesis. Calcif Tissue Int 86: 91-103.

19 Lagerstrom, M., N. Dahl, Y. Nakahori, Y. Nakagome, B. Backman, U. Landegren, U. Pettersson (1991) A deletion in the amelogenin gene (AMG) causes X-linked amelogenesis imperfecta (AIH1). Genomics 10: 971-975.

20 Lee, S.K., J.C. Hu, J.D. Bartlett, K.E. Lee, B.P Lin, J.P. Simmer, J.W. Kim (2008) Mutational spectrum of FAM83H: the C-terminal portion is required for tooth enamel calcification. Hum Mutat 29: E95-E99.

21 Lee, S.K., F. Seymen, H.Y. Kang, K.E. Lee, K. Gencay, B. Tuna, J.W. Kim (2010) MMP20 hemopexin domain mutation in amelogenesis imperfecta. J Dent Res 89: 46-50.
22 Lyaruu, D.M., A.L. Bronckers, L. Mulder, P. Mardones, J.F. Medina, S. Kellokumpu, R.P. Oude Elferink, V. Everts (2008) The anion exchanger Ae2 is required for enamel maturation in mouse teeth. Matrix Biol 27: 119127.

23 Moffatt, P., C.E. Smith, R. St-Arnaud, A. Nanci (2008) Characterization of Apin, a secreted protein highly expressed in tooth-associated epithelia. J Cell Biochem 103: 941956.

24 Nagano, F., H. Kawabe, H. Nakanishi, M. Shinohara, M. Deguchi-Tawarada, M. Takeuchi, T. Sasaki, Y. Takai (2002) Rabconnectin-3, a novel protein that binds both GDP/GTP exchange protein and GTPase-activating protein for Rab3 small $G$ protein family. The J Biol Chem 277: 9629-9632.

25 Ng, F.K., L.B. Messer (2009) Dental management of amelogenesis imperfecta patients: a primer on genotype-phenotype correlations. Pediatr Dent 31: 20-30.

26 Nylen, M.U., E.D. Eanes, K.A. Omnell (1963) Crystal growth in rat enamel. J Cell Biol 18: 109-123.

27 Okumura, R., Y. Shibukawa, T. Muramatsu, S. Hashimoto, K. Nakagawa, M. Tazaki, M. Shimono (2010) Sodium-calcium exchangers in rat ameloblasts. J Pharmacol Sci 112: 223-230.

28 Ozdemir, D., P.S. Hart, O.H. Ryu, S.J. Choi, M. Ozdemir-Karatas, E. Firatli, N. Piesco, T.C. Hart (2005) MMP20 active-site mutation in hypomaturation amelogenesis imperfecta. J Dent Res 84: 1031-1035.

29 Paine, M.L., M.L. Snead, H.J. Wang, N. Abuladze, A. Pushkin, W. Liu, L.Y. Kao, S.M. Wall, Y.H. Kim, I. Kurtz (2008) Role of NBCe1 and AE2 in secretory ameloblasts. J Dent Res 87: 391-395.

-30 Papagerakis, P., H.K. Lin, K.Y. Lee, Y. Hu, J.P. Simmer, J.D. Bartlett, J.C. Hu (2008) Premature stop codon in MMP20 causing amelogenesis imperfecta. J Dent Res 87: 56-59.

- 31 Porto, I.M., J. Merzel, F.B. de Sousa, L. Bachmann, J.A. Cury, S.R. Line, R.F. Gerlach (2009) Enamel mineralization in the absence of maturation stage ameloblasts. Arch Oral Biol 54: 313-321. 
32 Rajpar, M.H., K. Harley, C. Laing, R.M. Davies, M.J. Dixon (2001) Mutation of the gene encoding the enamel-specific protein, enamelin, causes autosomal-dominant amelogenesis imperfecta. Hum Mol Genet 10: 16731677.

-33 Sasaki, S., T. Takagi, M. Suzuki (1991) Cyclical changes in $\mathrm{pH}$ in bovine developing enamel as sequential bands. Arch Oral Biol 36: $227-231$.

- 34 Shore, R.C., B. Backman, S.J. Brookes, J. Kirkham, S.R. Wood, C. Robinson (2002) Inheritance pattern and elemental composition of enamel affected by hypomaturation amelogenesis imperfecta. Connect Tissue Res 43: 466-471.

35 Simmer, J.P., J.C. Hu (2002) Expression, structure, and function of enamel proteinases. Connect Tissue Res 43: 441-449.
36 Simmer, J.P., Y. Hu, R. Lertlam, Y. Yamakoshi, J.C. Hu (2009) Hypomaturation enamel defects in Klk4 knockout/LacZ knockin mice. J Biol Chem 284: 19110-19121.

37 Skobe, Z. (2006) SEM evidence that one ameloblast secretes one keyhole-shaped enamel rod in monkey teeth. Eur J Oral Sci 114(suppl 1): $338-342$.

38 Smith, C.E. (1998) Cellular and chemical events during enamel maturation. Crit Rev Oral Biol Med 9: 128-161.

39 Smith, C.E., A. Nanci (1995) Overview of morphological changes in enamel organ cells associated with major events in amelogenesis. Int J Dev Biol 39: 153-161.

40 Tsuchiya, M., R. Sharma, C.E. Tye, T. Sugiyama, J.D. Bartlett (2009) Transforming growth factor- $\beta 1$ expression is up-regulated in maturation-stage enamel organ and may induce ameloblast apoptosis. Eur J Oral Sci 117: 105-112.

41 Warshawsky, H., C.E. Smith (1974) Morphological classification of rat incisor ameloblasts. Anat Rec 179: 423-446.
42 Witkop, C.J., Jr. (1988) Amelogenesis imperfecta, dentinogenesis imperfecta and dentin dysplasia revisited: problems in classification. J Oral Pathol 17: 547-553.

43 Wright, J.T., T.C. Hart, P.S. Hart, D. Simmons, C. Suggs, B. Daley, J. Simmer, J. Hu, J.D. Bartlett, Y. Li, Z.A. Yuan, W.K. Seow, C.W. Gibson (2009) Human and mouse enamel phenotypes resulting from mutation or altered expression of AMEL, ENAM, MMP20 and KLK4. Cells Tissues Organs 189: 224-229.

44 Yang, X., L. Wang, Y. Qin, Z. Sun, Z.J. Henneman, J. Moradian-Oldak, G.H. Nancollas (2010) How amelogenin orchestrates the organization of hierarchical elongated microstructures of apatite. J Phys Chem B 114: 2293-2300. 